\title{
Determinants of pain and discomfort during mammography
}

\section{Determinantes da dor e desconforto durante a Mamografia}

Rita Menano Alves, Sónia Pinto Santos and Paula Jacinto

ERISA - Escola Superior de Saúde Ribeiro Sanches, Rua do Telhal aos Olivais n 8 - 8A, 1900-693 Lisboa

E-mail:ritamenanoalves@gmail.com

\begin{abstract}
Mammography is the main complementary exam for the breast cancer early diagnosis. However, discomfort and pain are frequent complaints during the exam.

Objective: to disclose discomfort and pain inflicted by the mammography and identify the determinants that might be related to thesesymptoms.

Methods: descriptive and transversal study involving 100 patients from public and private institutions of the larger Lisbon area. Patients answer to two questionnaires before and after the exam, to quantify pain and discomfort during the mammography, and find eventual symptoms related determinants.

Results: anxiety felt before the exam, previous mastalgia, discomfort expectations when the radiographer touches the breast, and pain expectations were the most relevant variables influencing the exam.

Conclusion: in general, the analyzed data concurs with the literature, with the exception of results referred to the use of oral contraceptive drugs.
\end{abstract}

Keywords: breast, mammography, pain, discomfort.

\section{Resumo}

A Mamografia é o principal meio complementar de díagnóstico precoce do cancro da mama. No entanto, o desconforto e a dor săo queixas verificadas frequentementeduranteo exame.

Objectivo: Conhecer o desconforto e dor ocasionados pela realização da mamografia e identificar os determinantes que possam estar relacionados com estes sintomas.

Métodos: Estudo descritivo e transversal incluindo 100 utentes real lizado em ambiente público e privado da área da grande Lisboa. Antes e após a realização do exame as pacientes responderam respectivamente a dois questionários, que permitiram quantificar a dor e desconforto durante a mamografia, e encontrar os possiveis determinantes relacionados com estes sintomas.

Resultados: As variáveis que mais influenciam a dor e o desconforto sentidos durante a realização do exame são a ansiedade sentida antes do exame, a existência de mastalgia prévia, as expectativas em relação ao desconforto à exposição das mamas, expectativas em relação ao descon forto ao toque nas mamas por parte do $\mathrm{TR}$, expectativas quanto à dor.

Conclusão: De um modo geral, os dados analisados concordam com a revisão da literatura, com a excepção dos resultados que dizem respeito à utilização decontraceptivos orais.

Palavras-chave: Mama, Mamografia, dor, desconforto. 


\section{Introduction}

Mammography is the main diagnosis exam used for the early detection of breast cancer, the most frequent tumor and the first cause of death by cancer in women all over the world ${ }^{[1]}$. The incidence and the favorable prognosis makes breast cancer the one with higher prevalence ${ }^{17 \mathrm{I}}$ In Portugal, breast cancer is the most frequent tumor in women, being responsible for $17 \%$ of the total oncological deaths and the primary cause of death in women with tumors "it. Actually it is recommended that women over 35 years old have their first mammography. Beyond 50 's women should have their mammography annually ${ }^{(2)}$. The mammography implies the compression of the breast, with a force between 108 and $196 \mathrm{~N}$ (11 to $20 \mathrm{~kg}$ ), which might causes pain during the exam ${ }^{27}$. In every exam, breast compression varies with the need, having in mind the best image possible, depending on the patient's threshold. To obtain an adequate image for a good diagnosis, a precise careful breast position during the exam is imperative ${ }^{(2)}$. There are other relevant factors for a good diagnosis, such as the smallest breast thickness to visualize all the breast tissue, to reduce kinetic and geometric flou, and to reduce scattered radiation ${ }^{(2.5)}$. And these justify the compression.

Breast compression was identified as the main cause of pain and physical discomfort felt during the mammography ${ }^{(4)}$, being also associated to the patient's age ${ }^{(9)}$. Other potential associated factors during the exam are literacy, number of children, number of previous mammographies, RT (radiology technician) performance, service satisfaction ${ }^{[20)}$ and the use of oral contraceptive drugs ${ }^{\text {") }}$. The exam related anxiety was also related with the level of pain felt by the patient ${ }^{(3.12)}$. According to Menke et al, cyclic pain occurs in women from puberty up to 35 years of age, however it might occur later in menopause, ceasing when menopause starts. Thus it might be referred has one of the causes for pain in mammography ${ }^{\langle 13\}}$.

So, it's important to take actions to reduce the pain and discomfort associated with the exam, so that women can use this technology for their benefit, without suffering. This study's objectives aim to characterize the occurrence of pain and discomfort in the mammography, and identify the related determinants.

\section{Introdução}

A mamografia é o principal meio complementar de diagnóstico para a deteç̧ão precoce do cancro da mama, o tumor mais frequente e a primeira causa de morte por cancro em mutheres no mundo " $"$. A elevada incidência e o prognóstico favorável fazem com que o cancro da mama seja o tumor com maior prevalência ${ }^{(1)}$. Em Portugal, o cancro da mama है o tumor mais frequente em mulheres, responsável por $17 \%$ do total de mortes por causas oncológicas e a principal causa de morte em mulheres com neoplasias ${ }^{(1)}$. Actualmente, recomenda-se que, a partir dos 35 anos, todas as mulheres realizem a primeira mamografia. Com idades acima dos 50 anos, deverão submeter-se a mamografia anualmente ${ }^{(2)}$.

A realização da mamografia implica a compressão da mama, com uma força entre 108 a $196 \mathrm{~N}$ (11 a $20 \mathrm{~kg}$ ), podendo esta força provocar dor durante a realização do exame ${ }^{[2]}$. Em todos os exames, a compressão da mama é realizada consoante a necessidade, tendo como principal objectivo obter-se a melhor imagem possivel, mas tendo sempre em conta a forma como cada paciente suporta a compressão. Para obter uma imagem adequada para um bom diagnóstico, é imperativo o posicionamento preciso $\mathrm{e}$ cuidadoso da mama durante o exame ${ }^{(2)}$. Existem outros factores relevantes para um bom diagnóstico, tais como a diminuição da espessura mamária para visualização de todo o tecido mamário, a diminuição do flou cinético e geométrico, e a redução da radiação difusa ${ }^{0.6}$, razões pelas quais se realiza a compressäo.

A compressão da mama foi identificada como a principal causa de dor e desconforto físico sentidos durante a mamografia ${ }^{(4)}$, estando estes também associados à idade da

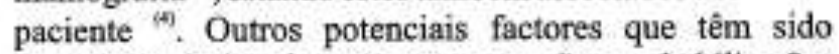
associados à dor durante o exame são: as habilitaçōes literárias, o número de filhos, número de mamografias anteriores, atendimento por parte do Técnico de Radiologia (TR), a satisfação com o serviço ${ }^{[20)} \mathrm{e}$ ainda a utilização de contraceptivos orais (7). A ansiedade provocada pelo exame também já foi relacionada com o grau de dor sentido pela paciente ${ }^{(x-12)}$. Segundo Menke et al, a mastalgia cíclica acontece a mulheres da puberdade aos 35 anos de idade, porém pode ocorrer mais tardiamente, na pós-menopausa, e costuma cessar quando se instala a menopausa. Por isso esta pode ser referenciada como uma das causas de dor durante a mamografia ${ }^{(1)}$.

Torna-se, desta forma, importante, tomar medidas para a diminuição da dor e do desconforto associados ao exame de mamografia, de maneira a que a mulher possa usufruir dessa tecnologia para seu beneficio, mas sem sofrimento associado.

Este estudo tem como objectivos caracterizar a ocorrência de dor e desconforto aquando da realização da mamografia e identificar os determinantes que possam estar associados a estes sintomas. 


\section{Methodology}

A transversal descriptive study was applied to a convenient sample of 100 patients, from which 50 had their exam in a private institution (equipment: Siemens Mammomat 300 , with a compression force $147 \mathrm{~N}$ ) and 50 in two public institutions (equipments: GE Senographe DMR and Bennet Contout, with compression forces 98 to $117 \mathrm{~N})$. After checking in, but before the exam, patients were asked to answer to an initial questionnaire involving demographic questions and their expectations to the pain and discomfort of the exam. The second questionnaire, presented after the exam, included questions about the pain and discomfort actually felt during the exam. Both questionnaires were replied by the authors in the institution's waiting room's during the months of March and April 2009.

To evaluate the pain and discomfort symptoms, a quantitative scale was adapted from the Circular Normativa n 09 / Direç̧ão Geral da Saúde ${ }^{(14)}$. Patients were asked to classify their expectations regarding pain and discomfort, and the intensity of the pain and discomfort of the exam as well, according to a 1 to 5 scale, being: 1-no pain/no discomfort, 2-slight pain/ discomfort, 3 - moderated pain/discomfort, 4 intense pain/discomfort and 5 - maximum pain/discomfort.

Data were analised by a SPSS 14.0 platform, using the Qui-Square Test and/or the Pearson Correlation test, and a $95 \%$ level of confidence adopted.

\section{Results}

The sample inluded 100 women from 28 to 83 years old (mean: $55,4 \pm 13,2$ years), with a mode of 69 years old. Regarding the academic qualifications, these were distributed from "no education" to "higher education", having two modes, on in the primary school $(36 \%)$ and the other in college level $(28 \%)$ (Figure 1). In the total sample, $55 \%$ of the patients were asymptomatic, most of them had children ( $81 \%)$, and $71 \%$ breastfed. The use of oral contraceptive pills was reported by $21 \%, 38 \%$ were in menopause and $13 \%$ submitted to Hormone ReplacementTherapy (HRT), Family history of breast disease was reported by $27 \%$ and only $13 \%$ were having their first mammography. In this sample, $36 \%$ reported previous mastalgia and $70 \%$ said that felt comfortable in the exam(Figure 1).

\section{Metodologia}

Realizou-se um estudo descritivo transversal, com uma amostra de conveniência de 100 pacientes, das quais 50 realizaram o exame numa instituição privada (equipamento: Siemens Mammomat 300, com uma força de compressão $\approx 147 \mathrm{~N}$ ) e 50 em đuas instituições públicas (equipamentos: GE Senographe DMR e Bennet Contour, com uma força de compressão de $\approx 98$ a $117 \mathrm{~N}$ ).

Aquando da chegada ao serviço e antes do exame, as pacientes responderam a um questionário inicial, com perguntas demográficas e acerca das suas expectativas em relação à dor e desconforto do exame. $\mathrm{O}$ segundo questionário, aplicado após a mamografia, incluia perguntas sobre os sintomas de dor e desconforto, de facto, sentidos durante o exame. Ambos os questionírios foram executados pelas autoras na sala de espera do estabelecimento, durante os meses de Março e Abril de 2009.

Para a avaliação dos sintomas de dor e desconforto adaptou-se uma escala quantitativa da Círcular Normativa ñ09/Direcção-Geral da Saúde ${ }^{064}$. Assim, solicitou-se às doentes que classificassem as suas expectativas relativamente à dor e desconforto, e à intensidade de dor $\mathrm{e}$ desconforto sentidos aquando da realizaçăo do exame, segundo uma escala de 1 a 5 , com a seguinte correspondência: 1-sem dor/sem desconforto, 2dor/desconforto ligeira(o), 3- dor/desconforto moderada(o), 4- dor/desconforto intensa(o) e 5dor/desconforto máxima(o).

Os dados foram tratados em plataforma SPSS versão 14.0, com utilização do Teste do Qui-Quadrado e/ou Correlação de Pearson. Considerou-se uma relação estatisticamente significativa quando $\mathrm{p}$ foi igual ou inferior a 0.05 , com um intervalo de confiança de $95 \%$.

\section{Resultados}

A amostra compreendeu 100 mulheres dos 28 aos 83 anos (média: 55,4 4 13,2 anos), com uma moda de 69 anos. Em relação às habilitações literárias, distribuiram-se desde a falta de instrução até ao ensino superior, verificando-se duas modas, uma ao nivel do ensino primário (36\%) e outra no ensino superior (28\%) (Ver Figura 1). Da totalidade da amostra, $55 \%$ das pacientes que realizou exame era assintomática, a maioria tinha filhos $(81 \%)$, tendo $71 \%$ amamentado. $\mathrm{O}$ uso de contraceptivos orais foi relatado por $21 \%$, encontravam-se em menopausa $38 \%$ e a realizavam Terapia Hormonal de Substituição (THS) 13\%. Antecedentes familiares de patologia mamária foram reportados por $27 \%$ e apenas $13 \%$ realizavam exame pela primeira vez. Nesta populaçāo, $36 \%$ auto-reportaram mastalgia prévia e $70 \%$ disseram sentir conforto quanto ao exame que iam realizar (Ver Figura 1). 


\begin{tabular}{|c|c|c|}
\hline \multirow{2}{*}{ Institution / Instituiçăo } & Public & $50 \%$ \\
\hline & Private & $50 \%$ \\
\hline \multirow{6}{*}{ Age / Idade } & 25.34 years/anos & $3 \%$ \\
\hline & $35-44$ years/anos & $23 \%$ \\
\hline & 45.54 years/anos & $23 \%$ \\
\hline & $55-64$ years/anos & $22 \%$ \\
\hline & $65-74$ years/anos & $19 \%$ \\
\hline & $? 75$ years/ancs & $10 \%$ \\
\hline \multirow{6}{*}{$\begin{array}{l}\text { Academic } \\
\text { Qualifications / } \\
\text { Habilotaçōes Literárias }\end{array}$} & No Studies/sem estudos & $2 \%$ \\
\hline & Pimary School/ensino primário & $36 \%$ \\
\hline & Prep School / ensino preparatónio & $10 \%$ \\
\hline & 39 Cido & $11 \%$ \\
\hline & 7th to 9 th Grade & $13 \%$ \\
\hline & College/University/Ensino Superior & $28 \%$ \\
\hline \multirow{2}{*}{$\begin{array}{l}\text { Type of Exam / Tipo de } \\
\text { Utente }\end{array}$} & Screening/Assintomática & $55 \%$ \\
\hline & Poutine / Sintomática & $45 \%$ \\
\hline \multirow{2}{*}{ Children / Filhos } & Yes/sim & $81 \%$ \\
\hline & No/não & $19 \%$ \\
\hline \multirow{2}{*}{ Breastfeed / Amamentaçăo } & Yes / sim & $71 \%$ \\
\hline & No/não & $29 \%$ \\
\hline \multirow{2}{*}{$\begin{array}{l}\text { Oral } \\
\text { Contraceptives / } \\
\text { Contracepção Oral }\end{array}$} & Yes/sim & $21 \%$ \\
\hline & No / nāo & $79 \%$ \\
\hline \multirow{2}{*}{ Menopause } & Yes/sim & $38 \%$ \\
\hline & No/ nāo & $62 \%$ \\
\hline \multirow{2}{*}{$\begin{array}{l}\text { Hormonal } \\
\text { Heplacement Therapy / } \\
\text { Terapia Hormonal de } \\
\text { Substituiçāo }\end{array}$} & Yes / sim & $13 \%$ \\
\hline & No/năo & $87 \%$ \\
\hline \multirow{2}{*}{$\begin{array}{l}\text { Family History / } \\
\text { Antecedentes } \\
\text { Familiares } \\
\end{array}$} & Yes/sim & $27 \%$ \\
\hline & No / nẳo & $73 \%$ \\
\hline \multirow{2}{*}{$\begin{array}{l}\text { First } \\
\text { Mammography / } 10 \\
\text { Mamografia } \\
\end{array}$} & Yes/sim & $13 \%$ \\
\hline & No / nåo & $87 \%$ \\
\hline \multirow{2}{*}{$\begin{array}{l}\text { Usual pain in the breast } \\
\text { /dorfrequente (mama) }\end{array}$} & Yes/sim & $36 \%$ \\
\hline & No / nắo & $64 \%$ \\
\hline \multirow{3}{*}{$\begin{array}{l}\text { Confort related to the } \\
\text { exam / conforto na } \\
\text { realizaçäo do exame }\end{array}$} & Yes/sim & $70 \%$ \\
\hline & No / nāo & $22 \%$ \\
\hline & Doesn't know / não sabe & B\% \\
\hline
\end{tabular}

Figure 1 - Demographic Characteristics of the Sample.

Figura 1- Caracterização Demográfica da Amostra.

For the 13 women having their first mammography, $69,23 \%(\mathrm{~N}=9)$ had a slight or moderated pain (Figure $3)$. In the rest of the group $(n=87)$ women who already experienced mammography, $50,57 \%(n=44)$ expected to have moderated pain, has it was shown in Figure 4. After the exam, from these 87 women. $51,72 \%(\mathrm{~N}=45)$ reported having slight or moderated pain, and $21 \%$
Comparando as expectativas de dor e a dor relatada, verificou-se que $20 \%$ não tinha qualquer expectativa de vir a ter sintomas de dor, no entanto $69 \%$ relata dor (Ver Figura 2). Das 13 mulheres que realizou mamografia pela primeira vez, $69,23 \%(\mathrm{~N}=9)$ tinha expectativas de dor ligeira ou moderada (Ver Figura 3). No conjunto das 87 que realizavam a mamografia subsequente, 
$(\mathrm{N}=18)$ complained of intense or maximum pain (Figure 4).
$50.57 \%(\mathrm{~N}=44)$ tinham a expectativa de ter dor ligeira ou moderada, como se apresenta na Figura 4. Após o exame, destas 87 mulheres, $51.72 \%(\mathrm{~N}-45)$ relatou ter tido dor ligeira ou moderada, e cerca de $21 \%(\mathrm{~N}=18)$ queixou-se de dor intensa ou máxima (Ver Figura 4).

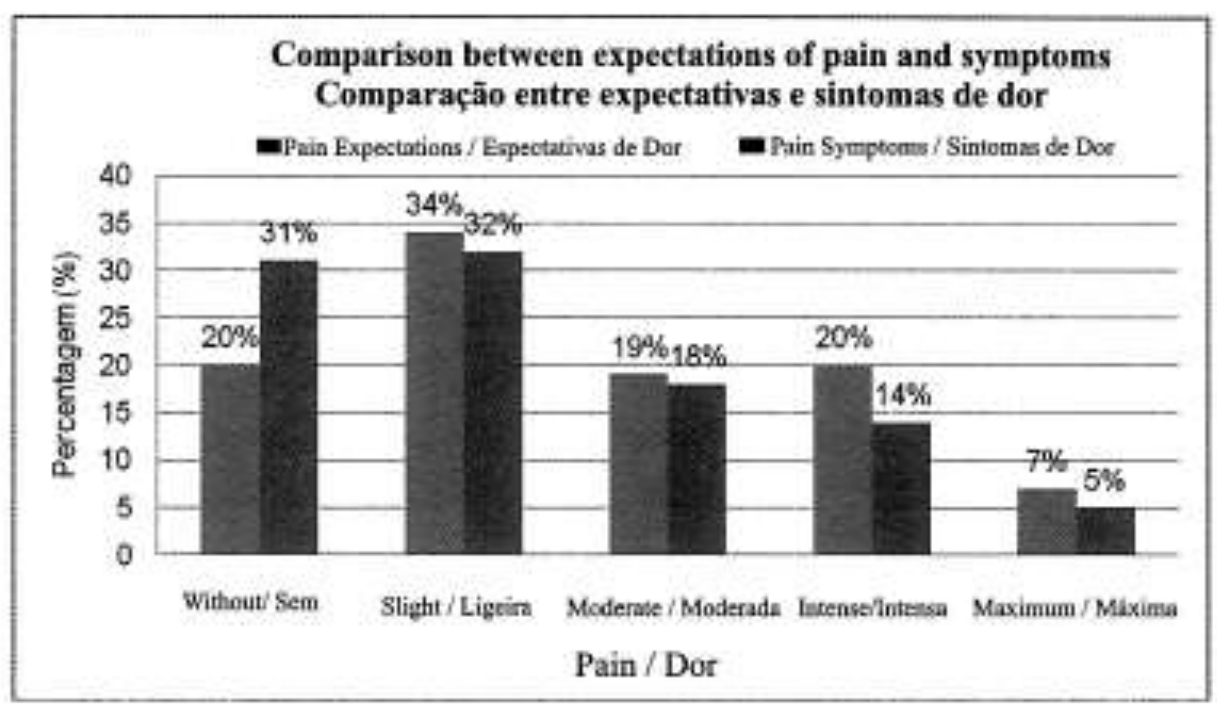

Figure 2 - Comparison between expectations of pain and symptoms

Figure 2 - Comparação entre expectativas e sintomas de dor

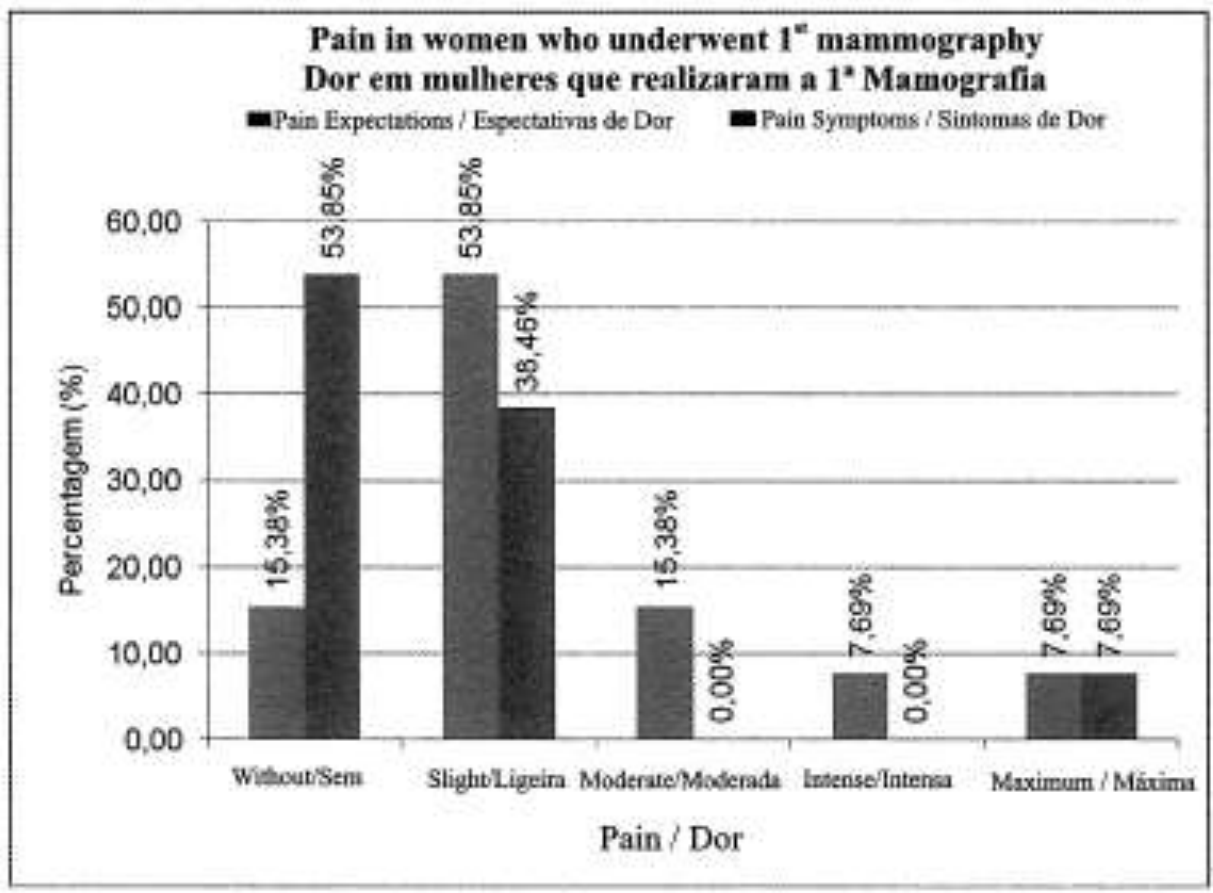

Figure 3 - Pain in women who underwent $1^{\text {r mammography }}$

Figura 3 - Dor em Mulheres que realizaram a $1^{2}$ Marnografia 


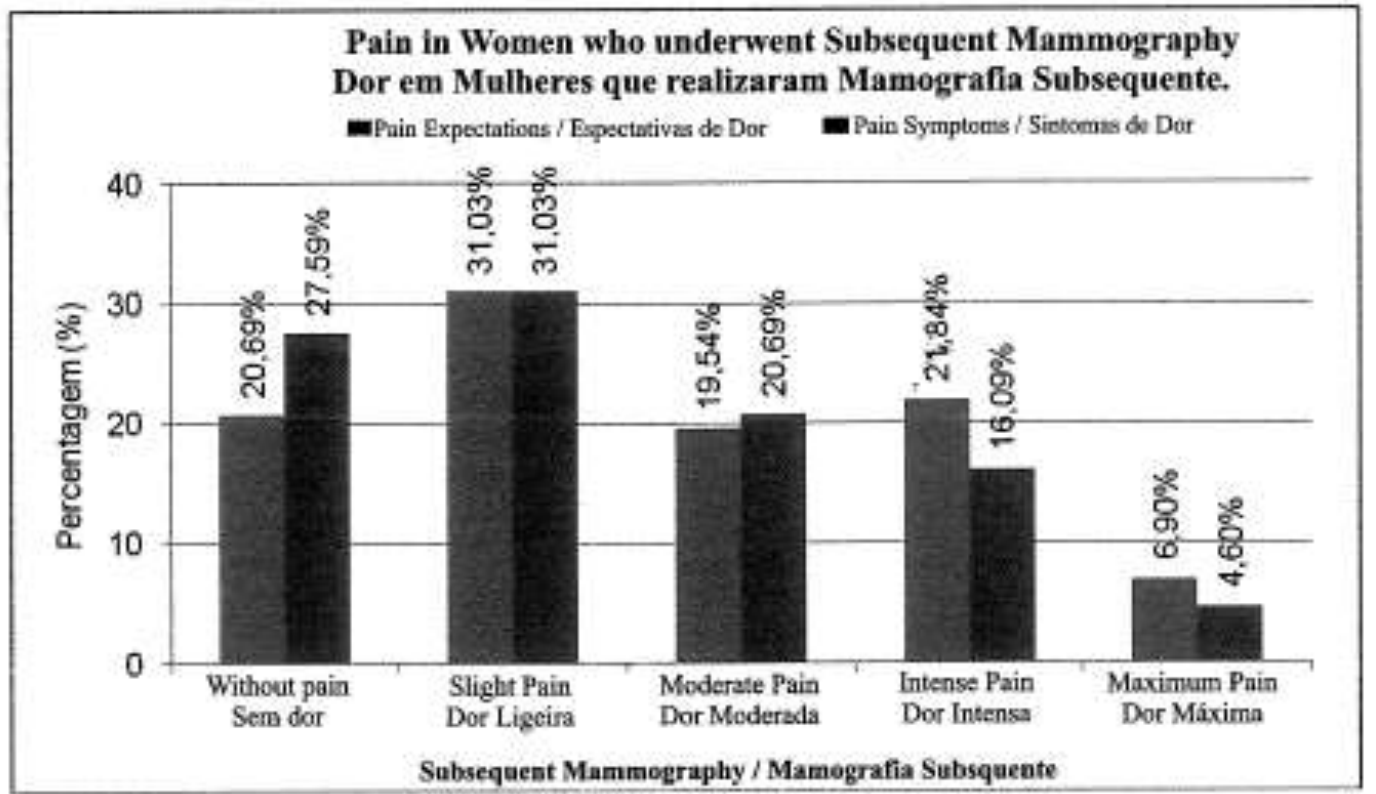

Figure 4 - Pain in Women who underwent Subsequent Mammography Figura 4.- Dor em Mulheres que realizaram Mamografia Subsequente.

In the mastalgia subgroup $(\mathrm{N}=64)$, only $31,2 \%(\mathrm{~N}=20)$ auto reported being anxious $(\mathrm{p}=0,034)$ (Figure 5). From the patients that didn't use oral contraceptives, $63 \%$ referred pain symptoms during the mammography $(p=0,013)$, from slight to maximum intensity (Figure 6).
No subgrupo de mulheres que referiu sofrer de mastalgia $(\mathrm{N}=36), 52,8 \%(\mathrm{~N}=19)$ estavam, tambem, ansiosas, enquanto que das restantes, sem mastalgia $(\mathrm{N}=64)$, apenas $31,2 \%(\mathrm{~N}=20)$ auto-reportou estarem ansiosas $(\mathrm{p}=0,034)$ (Ver Figura 5). Das pacientes que não realizavam contracepçåo oral $63 \%$ teve sintomas de dor durante a mamografia ( $\mathrm{p}=0.013)$, variando de intensidade ligeira a máxima (Ver Figura 6).

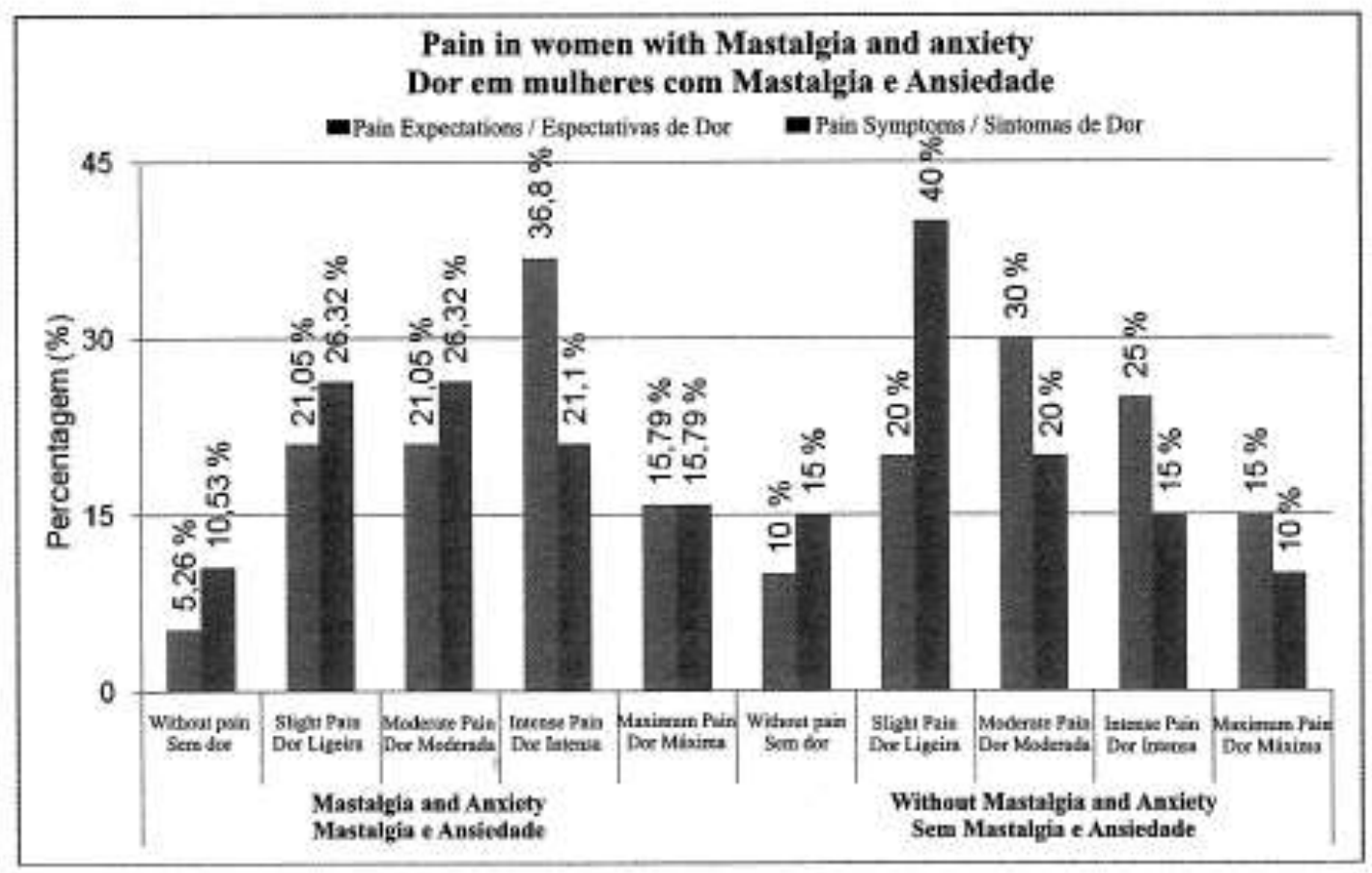

Figure 5 - Anxiety in Women with Mastalgia

Figura 5.- Ansiedade em Mulheres com Mastalgia. 


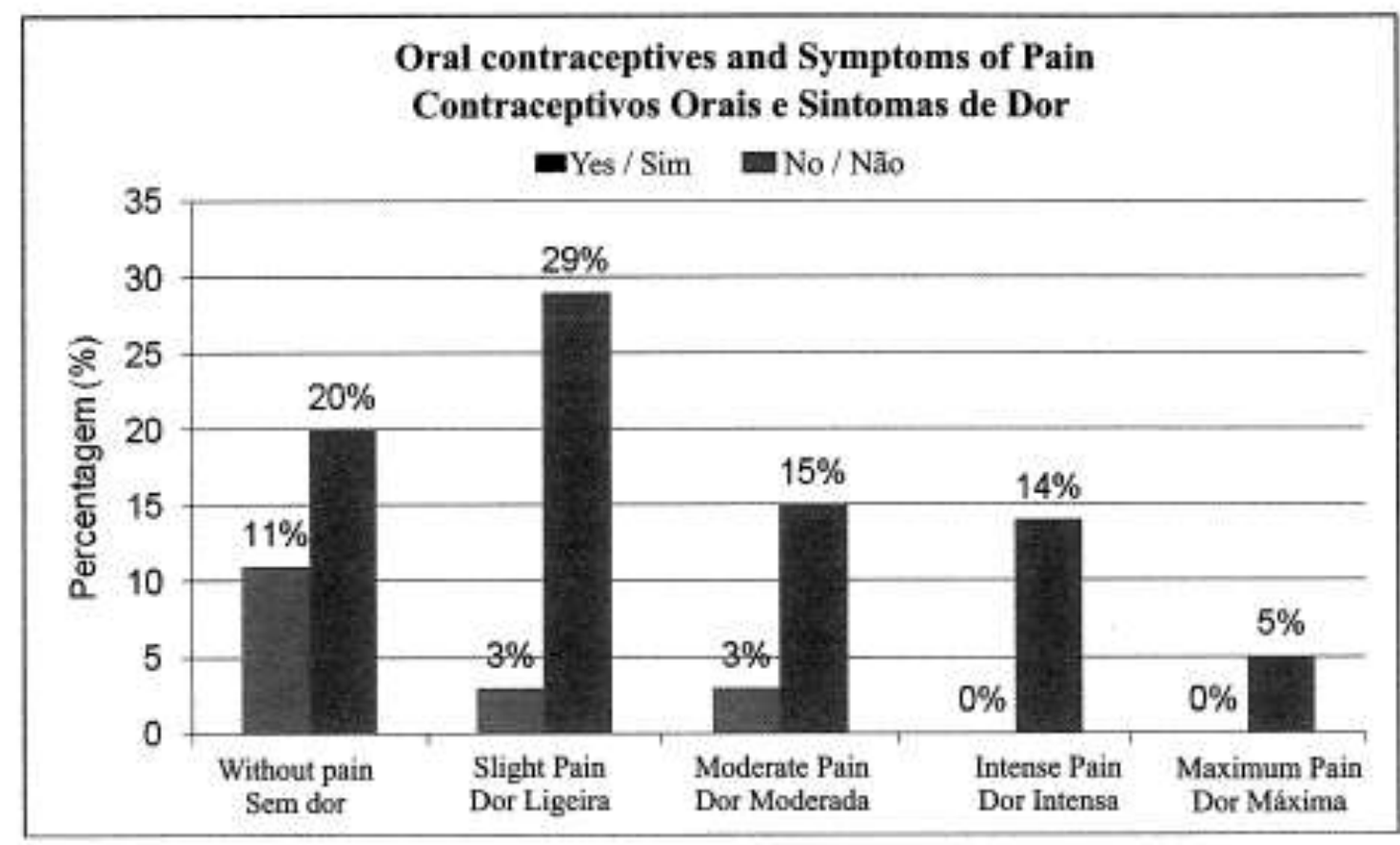

Figure 6 - Oral contraceptives and Symptoms of Pain

Figura 6. - Contraceptivos Orais e Sintomas de Dor.

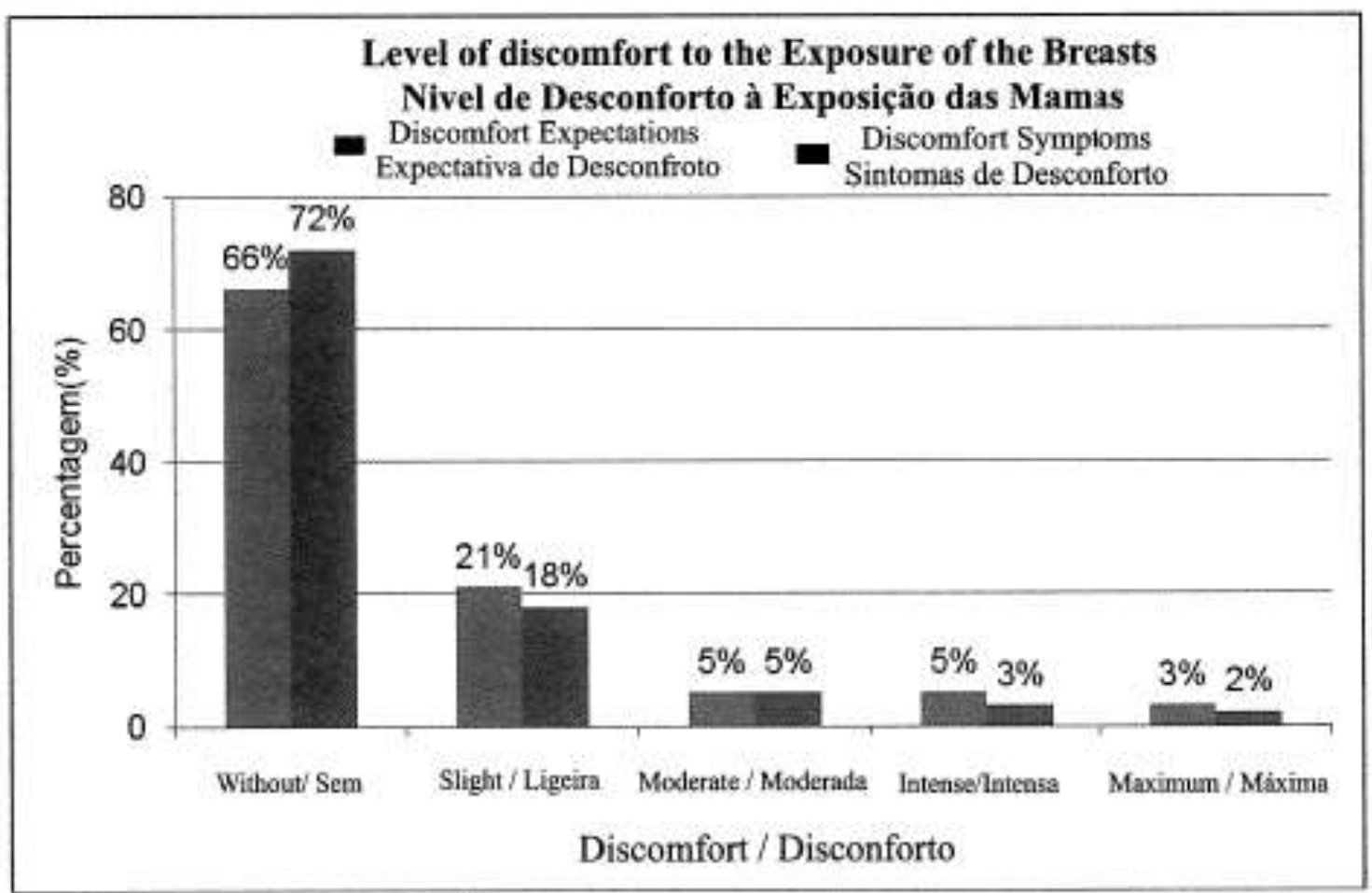

Figure 7 - Level of discomfort to the Exposure of the Breasts

Figura 7.- Nivel de Desconforto à Exposição das Mamas. 


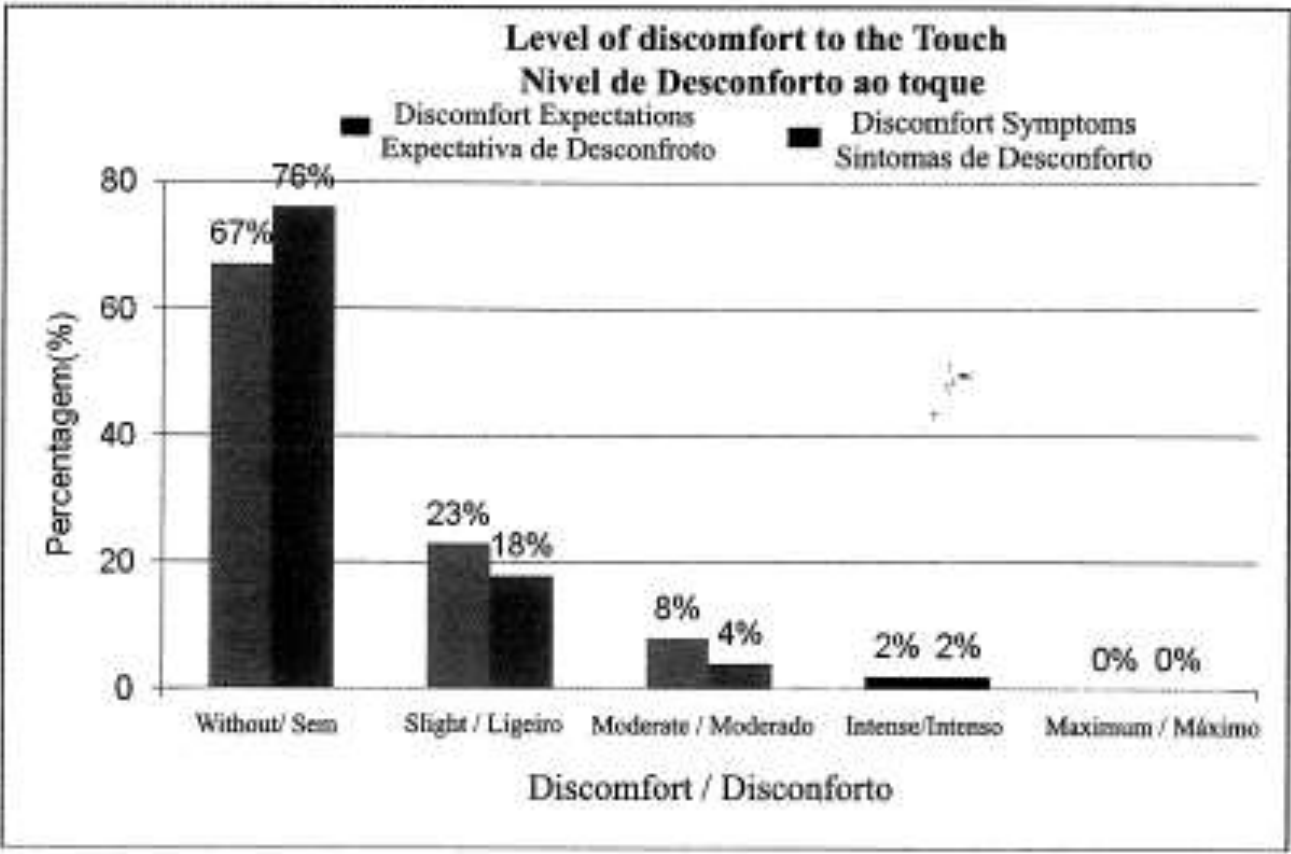

Figure 8 - Level of Discomfort to the Touch by the Radiographer Figura 8.- Nivel de Desconforto ao Toque do Técnico de Radiologia

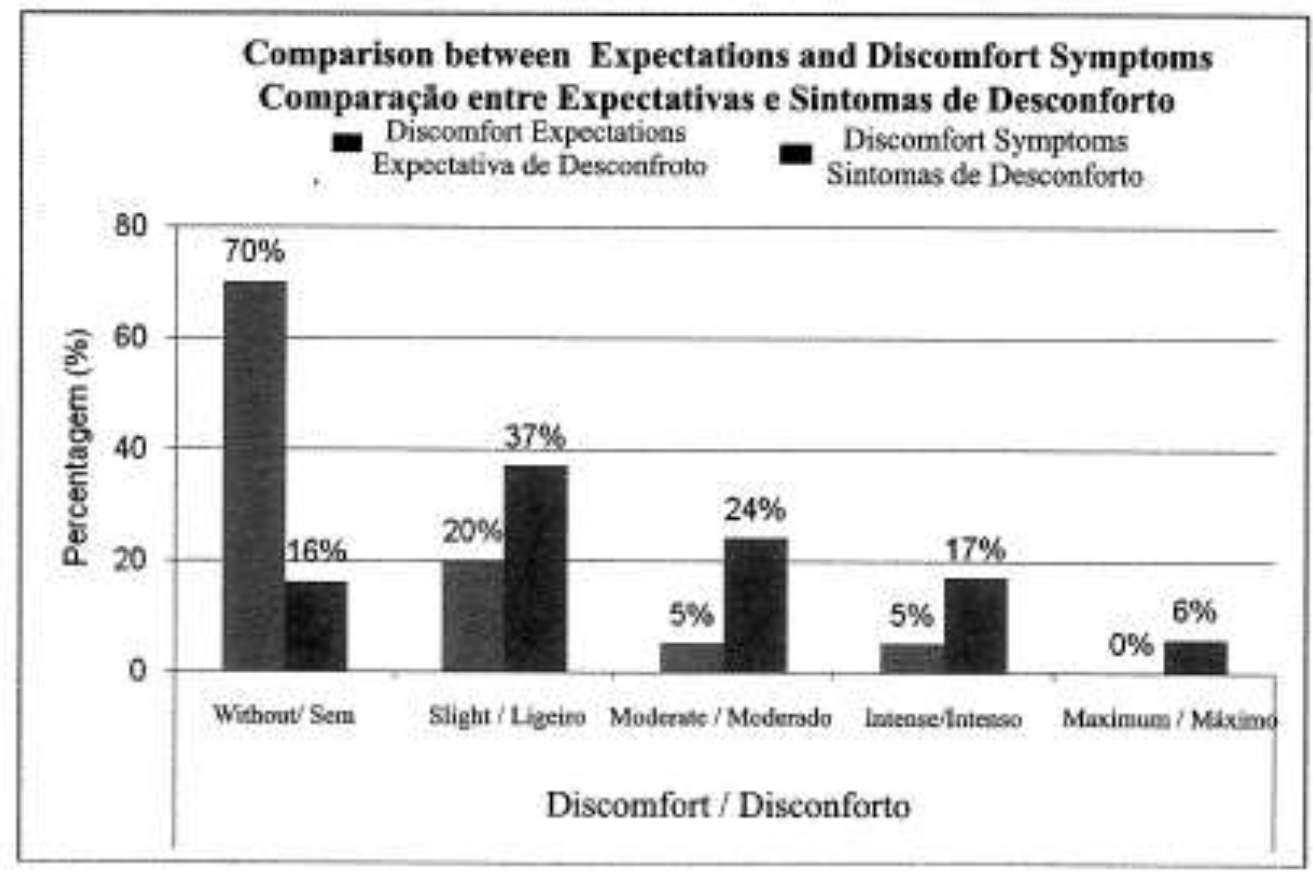

Figure 9 - Comparison between Expectations and Discomfort Symptoms Figura 9.- Comparação entre Expectativas de Desconforto e Sintomas de Desconforto

Discomfort expectations related with breasts exposure and touch by the technician can be found in Figures 7 and 8. Most of the women, $66 \%$, didn't have any no discomfort expectation by exposing their breasts, nor from being touched by the Radiographer in this context $67 \%$. In general, discomfort expectations and real discomfort felt during the exam (discomfort in the 82
As expectativas de desconforto quanto à exposição das mamas e ao toque do TR, assim como os sintomas de desconforto relatados encontram-se nas Figuras 7 e 8 , respectivamente. Pode verificar-se que a maioria das mulheres não teve qualquer expectativa nem sintoma de desconforto quanto à exposiçắo das mamas, $66 \%$ e ao toque do TR, $67 \%$. 
exposure of the breasts and to the touch of the Radiographer) are statistically different $(\mathrm{p}=0,001)$ (Figure 9), which may show that more than half of the women $(70 \%)$ didn't have any expectation regarding feeling discomfort.

The referred discomfort felt by the women during the exam presents a positive correlation with many variables, such as the nudity related discomfort, the touch discomfort by the radiographer, and other pain and pain symptoms expectations as described in Table 1. There was also some positive correlations for pain involving the patient type, the use of birth control pills, breasts exposure, the technician's touch to discomfort to the touch of the Radiographer, as presented in Table 2. The pain expectation turned out to show the strongest correlation with pain during the exam.

There wasn't a statistically significant relation between reported pain or discomfort during the examination and the following variables: symptomatic or asymptomatic patients, children given birth, having breastfed, menopause, do HRT, family history of breast pathology, to be its first mammography and, age group.
De uma maneira geral, as expectativas de desconforto, oo desconforto realmente sentido no decorrer do exame (desconforto à exposição das mamas e ao toque dos TR) apresentam uma diferença estatisticamente significativa $(\mathrm{p}=0,001)$ (Ver Figura 9), podendo constatar-se que mais de metade das mulheres $(70 \%)$ não tinha qualquer expectativa de vir a sentir desconforto.

$O$ desconforto que as mulheres referiram durante o exame apresenta uma correlação positiva com diversas variáveis, expectativa de desconforto à nudez, expectativas em relação ao desconforto ao toque nas mamas por parte do $\mathrm{TR}$, expectativas quanto à dor e sintomas de dor, tal como descrito na Tabela 1. Para a dor, também foram identificadas várias correlações positivas nas variáveis tipo de utente, uso de contracepção oral, expectativas quanto ao desconforto à exposiçåo das mamas, expectativas quanto ao desconforto ao toque nas mamas por parte do TR, expectativas quanto à dor, sintomas de desconforto quanto à exposiçąo das mamas, sintomas de desconforto quanto ao toque do TR e desconforto sentido, similarmente apresentadas na Tabela 2. A expectativa de dor revelou ser a correlação mais forte com o relato de dor durante o exame. Não se verificou uma relação estatisticamente significativa entre relatar dor ou desconforto durante o exame e as seguintes variáveis: utentes sintomáticas ou assintomáticas, ter filhos, ter amamentado, encontrar-se em menopausa, realizar THS, antecedentes familiares de patologia mamária, primeira mamografia e classe etária.

Table 1- Positive correlations between the reported discomfort during the examination and others

Tabela 1. - Correlaçōes Positivas entre o Desconforto relatado durante o exame e outras

\begin{tabular}{|c|c|c|c|}
\hline \multicolumn{2}{|c|}{$\begin{array}{l}\text { Positive correlation between: } \\
\text { Correlaçåo Positiva entre: }\end{array}$} & $p$ & $\mathrm{P}$ (Pearson) \\
\hline $\begin{array}{c}\text { Reported } \\
\text { discomfort } \\
\text { during } \\
\text { examination: }\end{array}$ & $\begin{array}{l}\text { Expectation of nudity and } \\
\text { Expectativa de nudez e }\end{array}$ & 0.000 & 0.532 \\
\hline $\begin{array}{l}\text { Desconforto } \\
\text { relatado durante } \\
\text { exame: }\end{array}$ & $\begin{array}{l}\text { Touch Expectations by the } \\
\text { Radiographer and } \\
\text { Expectativas em relação ao toque } \\
\text { por parte do TR e }\end{array}$ & 0,000 & 0,612 \\
\hline & $\begin{array}{l}\text { Pain Expectations and } \\
\text { Expectativas quanto à dor e } \\
\text { Pain Symptoms } \\
\text { Sintomas de dor }\end{array}$ & 0,001 & 0,329 \\
\hline
\end{tabular}

For the patients who reported no pain, it appears that before the exam, $20 \%$ had no expectations of pain, whereas $53 \%$ expected slight to moderate pain and
Relativamente às utentes que referem não sentir dor, verifica-se que antes da realização do exame, $20 \%$ não tinham expectativas de sentir dor, enquanto que $53 \%$ 
$27 \%$ maximum pain. After the exam $31 \%$ didn't referred pain symptoms and $50 \%$ referred slight to moderate pain. (Figure 2). tinham expectativas de dor ligeira a moderada e $27 \%$ de dor intensa a máxima. Após a realização do exame 31\% não refere sintomas de dor e $50 \%$ dor ligeira a moderada. (figura 2)

Table 1 - Positive correlations between the pain reported during the examination and other variables.

Tabela 2. - Correlações positivas entre a Dor relatada durante o exame e outras variávcis.

\begin{tabular}{|c|c|c|c|}
\hline \multicolumn{2}{|c|}{$\begin{array}{l}\text { Positive correlation between: } \\
\text { Correlação Positiva entre: }\end{array}$} & \multirow{2}{*}{$\begin{array}{l}p \\
0,008\end{array}$} & \multirow{2}{*}{$\begin{array}{c}\mathrm{P} \text { (Pearson) } \\
0,262\end{array}$} \\
\hline $\begin{array}{l}\text { Reported pain } \\
\text { during }\end{array}$ & $\begin{array}{l}\text { Type of Patients and } \\
\text { Tipo de utente e }\end{array}$ & & \\
\hline \multirow[t]{7}{*}{$\begin{array}{l}\text { Dor relatada } \\
\text { durante exame: }\end{array}$} & $\begin{array}{l}\text { Oral contraceptives and } \\
\text { Contraceptivos orais e }\end{array}$ & 0,003 & 0,294 \\
\hline & $\begin{array}{l}\text { Discomfort about the nudity and } \\
\text { Desconforto face à nudez } \mathrm{e}\end{array}$ & 0,039 & 0,207 \\
\hline & $\begin{array}{l}\text { Discomfort to theTouch by the } \\
\text { Radiographer and } \\
\text { Desconforto ao toque doTR e }\end{array}$ & 0,000 & 0,377 \\
\hline & $\begin{array}{l}\text { Expectations about pain and } \\
\text { Expectativas quanto à dor e }\end{array}$ & 0,000 & 0,725 \\
\hline & $\begin{array}{l}\text { Discomfort about the exposure and } \\
\text { Desconforto quanto a axposiçäo e }\end{array}$ & 0,000 & 0,418 \\
\hline & $\begin{array}{l}\text { Discomfort about the Touch by the } \\
\text { Radiographer and } \\
\text { Desconforto quanto ao toque do TR e }\end{array}$ & 0,000 & 0,370 \\
\hline & $\begin{array}{l}\text { discomfort felt } \\
\text { Desconforto sentido }\end{array}$ & 0,000 & 0,413 \\
\hline
\end{tabular}

\section{Diseussion}

The existence of previous mastalgia was reported as a pain / discomfort related cause of anxiety, that may well be related to the reported fear of the examination resulting in women's pain expectation. Most of the patients using oral contraceptives referred no pain during mammography. This is contradicted by the study of Ruffo Jr. et al ${ }^{135}$ who referred that oral contraception leads to greater breast tenderness, increasing the pain. Moreover, most women using oral contraceptives referred no pain symptoms.

Patients with pain and discomfort expectations were

\section{Discussão}

A existência de mastalgia prévia foi referida como uma das causas de ansiedade em relação à dor ou desconforto, o que pode estar relacionado com o receio das mulheres do resultado do exame e as expectativas relativamente à dor. A maioria das pacientes que não utilizava contraceptivos orais teve sintomas de dor durante a mamografia. Isto vai em contra ao estudo de Ruffo Júnior et al ${ }^{195}$ que diz que a contracepção oral induz uma maior sensibilidade mamária, aumentando a dor. Por outro lado, a maioria das mulheres que realizava contracepção oral não teve sintomas de dor. 
those who have shown more pain symptoms. These expectations may be due to past experiences or information provided by friends/family, as found in a study by B. Davey".

Combination of discomfort expectation and symptoms regarding breasts exposure and the technician's touch was expected, taking into account all the psychological component involved in the examination. As reported by B. Davey ${ }^{77}$ the shame often felt by women regarding the nudity, and fear of the results verdict may be linked to breast cancer. The relationship between pain expectation and experienced discomfort during breasts exposure also shows a psychological connotation, which may be related to anxiety and nervousness expressed by woman just before exam, as published in previous studies ${ }^{[T-12 \%}$,

No relationship could be found between HRT and pain symptoms as published before ${ }^{(15)}$, suggesting that the increase in breast density caused by HRT reduces pain symptoms. The relationship between age and pain / discomfort symptoms referred by R. Gupta ${ }^{(3)}$, could not be found, following conclusion by $\mathrm{C}$. Wiratkapun et al $^{\text {a) }}$

Pain expectations before the exam influences the pain reported by patients during the mammography, which is consistent with other previous studies ${ }^{\text {कon }}$.

In terms of relevant studies for the future, it is suggested to increase the sample size in order to consolidate, confirming or not, results. It would be interesting to include the numerical values of the compression forces used in each examination in order to better identify pain / discomfort determinants. The occasional presence of students was not taken into account, But it may have influenced the result as the discomfort levels felt by the patients.

\section{Conclusions}

Mammography is a diagnostic tool that involves a high psychological component, so that, in addition to physical determinants such as mastalgia, prior anxiety and discomfort and pain expectations, often reported by patients, may greatly influence the levels of discomfort and pain felt during the exam.

Some factors that were not assessed and may have influenced the pain and discomfort symptoms were the type of equipment used, the compression exerted, and the occasional presence of students during the data collection period. The use of a small sample doesn't allow these data to be extrapolated to the general population and may have influenced some of the results, which ended up not coinciding with previously published studies.

The pain referred by patients during the exam, has shown significant correlations with several variables,
Pacientes com expectativas de dor e desconforto foram aquelas que mais tiveram sintomas de dor. Estas expectativas podem dever-se a experiências anteriores ou informaçōes fornecidas por amigos/familiares, tal como verificado num estudo realizado por B. Davey ${ }^{13}$. A associação de expectativas de desconforto na exposição das mamas e ao toque do TR com os sintomas de desconforto sentidos era de esperar, tendo em conta toda a componente psicológica que envolve o exame, como a vergonha geralmente sentida pela mulher relativamente à nudez $\mathrm{e}$ o medo do resultado que pode estar associado ao cancro da mama, como relatado por B. Davey ${ }^{\text {} 7}$. A relação das expectativas de dor com o desconforto sentido aquando da exposição das mamas tem também uma conotação psicológica, que pode estar relacionada com a ansiedade e nervosismo sentido pela mulher previamente à realização do exame, tal como constatado $\mathrm{cm}$ estudos anteriores $^{(\pi-12)}$

Năo se encontrou relação entre a realizaçāo de THS e sintomas de dor, ao contrário de outro estudo ${ }^{(16)}$, onde referem que o aumento da densidade mamária provocada pela THS leva a sintomas de dor. Tal como no estudo realizado por C. Wiratkapun et al ${ }^{13)}$ não foi verificada qualquer relação entre $\mathrm{a}$ idade e sintomas de dor e desconforto, ao contrário do estudo realizado por R, Gupta ${ }^{\text {sit }}$.

As expectativas de dor antes do exame acabam por influenciar a dor referida pelas utentes aquando da realização do exame, o que vai ao encontro de estudos anteriores ${ }^{(1,5)}$.

Em termos de estudos futuros pertinentes, sugere-se que se alargue o número da amostra para consolidar este estudo, confirmando-se, ou não, os mesmos resultados. Teria interesse incluir os valores numéricos da força de compressão utilizada em cada exame de modo a melhor poder identificar os determinantes de dor e desconforto. A presença esporádica de estagiários não foi tida em conta, o que pode ter influenciado os resultados obtidos, podendo mesmo influenciar os níveis de desconforto sentidos pelas pacientes.

\section{Conclusões}

A mamografia é um exame que acarreta uma elevada componente psicológica, pelo que, para além de determinantes físicos como a mastalgia prévia, a ansiedade $e$ as expectativas de desconforto e dor relatados pelas pacientes influenciam muito os niveis de desconforto e dor sentidos durante o exame.

Alguns factores que não foram avaliados e que podem ter influenciado os sintomas de dor e desconforto foram o tipo de equipamento utilizado, a compressão exercida e a presença ocasional de estagiários, durante o periodo de recolha de dados.

A utilizaçăo de uma amostra pequena não permite que 
being specially strong in those patients who already expected the pain.

For the variable discomfort, reported during the examination, the strongest correlation was found in those patients who already had discomfort expectations to the technician's touch.

In general terms, the analyzed data agrees with the literature review, except for those results involving the oral contraception.

\section{Acknowledgements}

The authors specially thank to all patients who agreed to participate in the study, and to all institutions allowing the involved data collection.

Also to Mariana Alves Pereira and Carla Monteiro for the demonstrated support and availability. estes dados sejam extrapolados para a população em geral, podendo ter influenciado alguns dos resultados, que acabaram por não coincidir com estudos realizados anteriormente.

Quanto à variável dor referido pelas utentes durante 0 exame apresenta uma correlação significativa com diversas variáveis, sendo a mais forte a sentida pelas utentes que já tinham expectativas de dor.

Relativamente à variável desconforto relatado durante o exame, verifica-se que a correlaçăo mais forte é sentida pelas utentes que tinham expectativas de desconforto ao toque nas mamas por parte do TR.

De um modo geral, os dados analisados concordam com a revisão da literatura, excepto os resultados que dizem respeito às utentes que realizam contracepção oral.

\section{Agradecimentos}

Os autores agradecem especialmente a todas as pacientes que se disponibilizaram para a participação neste estudo, assim como às instituiçōes que permitiram a recolha de dados.

As Professoras Mariana Alves-Pereira e Carla Monteiro também pelos apoio e disponibilidade demosntrados.

\section{References / Referincias}

[1] Bastos J, Barros H, Lunet N. Evoluçân da mortalidade per cancro da mama en Portugal (19552002). Acta Med Port 2007; 20: 139-144

[2]. BeotragerKI. Tratado de Tecnica Radiológica Base Anatônica. 5 th ed. Rio de haseim (BR): Gusanaberakoogan; 2001 .

[3]. Wiratkapun C, Lertsithichas P, wibulpolpraset $B$, et al. Breast pain and service satisfaction doring digital mammozraphy J Med Assoc Thai 2006 89(11): $1864-1873$.

[4]. Jackson V, Lex A, Smith D. Patient isscomfort during screen-film mammography. Radiology 1988, $168(2): 421-42.3$

[5]. Gupta R, Nayas M, Khoursheed M, et al. Pain during ranmography: impact of breast patholovies and deosographic factors. Med Princ Pract 2003, 12 . 180-183

[6]. Rutter R, Nayak M, Kroursheed M, if al. Discomfort and pain during mammography: description, predicbon, and pervertion. BMJ 1992 , 305: 443-445
[7]. Davey a. Pain during mammography possible risk factors ind ways to alleviate pain. Radiography $2007,13: 229-234$

[B]. Aro A, Absetz-Ylastulo P, Ferols T, et at Pain ant discomfort duning mammography. Europese Journal of Cancer 1998, 32(10): 1674-1679.

[9]. Bruyninckx E, Mortelmans D, Van Goethem M. et al. Risk factoca of pain in mammographic sereening. Social Science \& Modicine 1999, 49: 933. 941.

[10]. Keeners-Gels M. Groenentijk R. Van Den Hewvel J, et al. Phin experienced by women attenting breat caneer seterning. Breast Cancer Research and Trearmest 2000, 60: 235-240.

[11]. Goothem M, Mortielmans D, Brusninckx E, et al Influesce of the raliegrapber on the pain felt turing mammoography. Eur Rodtiol 2003, 13: 2354-2339.

[12]. Mainiero M, Schepps B, Clements $N$, et al. Mammograpby - related anxiety: Effect of preprocederal patient education. Womens Health issuns $2001,11 / 2): 110-115$
[13]. Menke $\mathrm{CH}_{3}$ Biagus JV, Cavalheiro JA, Rabin ECt. Alteracoles funcionais benignas da mama. In: Franco JM. Mitstologia, formeşầo do especialista. Slo Pauloc Atbeevu, 77-85, 1997.

[14]. Direcesie-Geral da Saúde. A Dor come 5 " sinal vital - Registo sistemático da intensidade da dor Circular Normativa n09/DGCG. 2003, Ministério đa Saúde.

[15]. Jíniar R, Fion W, Ramas F, ef al. Desconforto e dor durante realizaçăo da Mamokratia. Ver Assoc Med Bras 2006, 52(5): 333-336

[16]. MeNichalas $M$, Heneghas J, Milner $M$, et al. Pain and increased manmognphic donsity in women receiving bormone replacemest therapy. ARJ 1994, 163:311-315. 